\title{
Inherited thrombophilia secondary to protein S deficiency associated with ischemic stroke. Case report
}

\author{
Diana Matcau', Claudia Barsan'1, Traian Flavius Dan'1 , Camelia Barbusi', \\ Any Docu Axelerad ${ }^{2}$, Dragos Catalin Jianu ${ }^{1}$ \\ ${ }^{1}$ Department of Neurology, "Victor Babes" University of Medicine and Pharmacy, Timisoara, Romania \\ 2Department of Neurology, "Ovidius" University of Medicine and Pharmacy, Constanta, Romania
}

\begin{abstract}
Thrombophilia is characterized by an increased tendency to arterial and venous thrombus formation. Inherited thrombophilia can be secondary to protein $\mathrm{S}$ deficiency. Concomitant use of oral contraceptive pills increases the risk of venous thromboembolism and arterial thrombosis. We report a case of a 34-year-old female who used oral contraceptive pills for many years, who developed acute ischemic stroke of right occipital lobe with secondary left superior homonymous quadrantanopia. We have run several tests to establish the ischemic stroke etiology: unenhanced brain and cervical spine computed tomography, brain magnetic resonance imaging and magnetic resonance angiography (including MR venography), computed tomographic angiography, echocardiography, extracranial duplex ultrasound and laboratory data (including thrombophilia testing). The laboratory investigations confirmed that the ischemic stroke of our patient resulted from a severe inherited thrombophilia secondary to protein S deficiency.
\end{abstract}

Keywords: ischemic stroke, thrombophilia, protein S, contraceptive pills

\section{INTRODUCTION}

In thrombophilia, there is an increased tendency to arterial and venous thrombus formation. Inherited thrombophilia includes deficiencies in the anticoagulant proteins such as antithrombin, protein $\mathrm{C}$, protein $\mathrm{S}$, activated protein $\mathrm{C}$ resistance, factor $\mathrm{V}$ Leiden mutation, prothrombin gene (G20210A) mutation; methylene-tetrahydrofolate reductase (MTHFR) gene mutation etc (1-3).

Protein $\mathrm{S}$ is a K-dependent anticoagulant protein, which undertakes the role of a non-enzymatic cofactor of the activated protein $\mathrm{C}$. In healthy individuals, about $30-40 \%$ of protein $\mathrm{S}$ is in a free state. Only the free form of protein $\mathrm{S}$ can be a cofactor to the protein $\mathrm{C}$ system. The prevalence of protein $\mathrm{S}$ deficiency in Caucasians is about $0.03 \%$ (4). Protein $\mathrm{S}$ deficiency may be inherited or acquired; the latter is usually due to hepatic diseases, a vitamin $\mathrm{K}$ deficiency or sickle cell anemia (4). Inherited protein $\mathrm{S}$ deficiency is caused by mutations in the PROS1 gene (autosomal dominant inheritance pattern) and can be divided into 3 types based on how these mutations affect protein S: type I protein $\mathrm{S}$ deficiency is a reduction in the level of free and total protein S, type II deficiency is a reduction in the cofactor activity of protein $\mathrm{S}$, with normal antigenic levels and type III deficiency is a reduction in the level of free protein S only (4).

The main clinical manifestations of protein $\mathrm{S}$ deficiency are represented by deep vein thrombosis, and pulmonary thromboembolism; a few cases of arterial thrombosis, most of them causing ischemic stroke (4), have been reported. Concomitant use of contraceptive pills increases the risk of venous thromboembolism and arterial thrombosis $(1,3)$.

\section{CASE REPORT}

We report the case of a 34-year-old woman who came in the Emergency Service of Timisoara Clin- 
ical Emergency County Hospital, in Ianuary 2016, accusing vertigo, nausea, vision problems, occipital headache (3/10) and posterior cervical pain. The symptoms started suddenly, one day before admission, after a sudden movement of the head. From the personal history of the patient, we retain: she had given birth twice, was diagnosed with chronic autoimmune thyroiditis (untreated at the moment of examination) and she had been using contraceptive pills, intermittently for about 15 years.

Neurological examination was positive for left superior homonymus quadrantanopia, and a slightly insecure gait, secondary to the vertigo.

In the Emergency Department, a native brain computed tomography (CT) scan (which revealed a hypo dense area localized in the right occipital lobe having a minimal mass effect) (Fig. 1) and a native cervical spine CT scan (which described a posterior alignment of the cervical vertebral bodies) were performed.

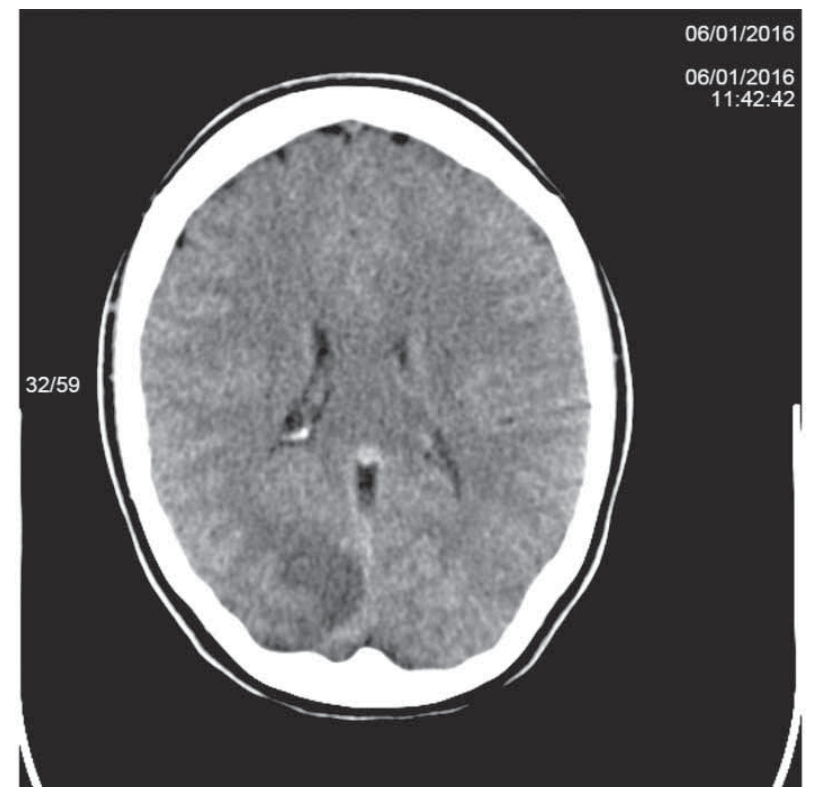

FIGURE 1. Native brain CT scan revealed a hypodense area localized in the right occipital lobe having a minimal mass effect.

The patient was admitted at the Neurology Department with the diagnosis of acute right occipital ischemic stroke.

In the same day, a brain magnetic resonance imaging (MRI) combined with MR angiography (MRA), including MR-venography were performed. MRI revealed a cortical/subcortical lesion partially involving the right occipital lobe (the calcarine fissure was affected) (hyper-signal T2 and FLAIR, slightly hypo-signal T1, no contrast uptake-suggesting an ischemic etiology for the lesion described) (Fig. 2 A and Fig. 2 C); MR-A images were normal (Fig. 2 B).

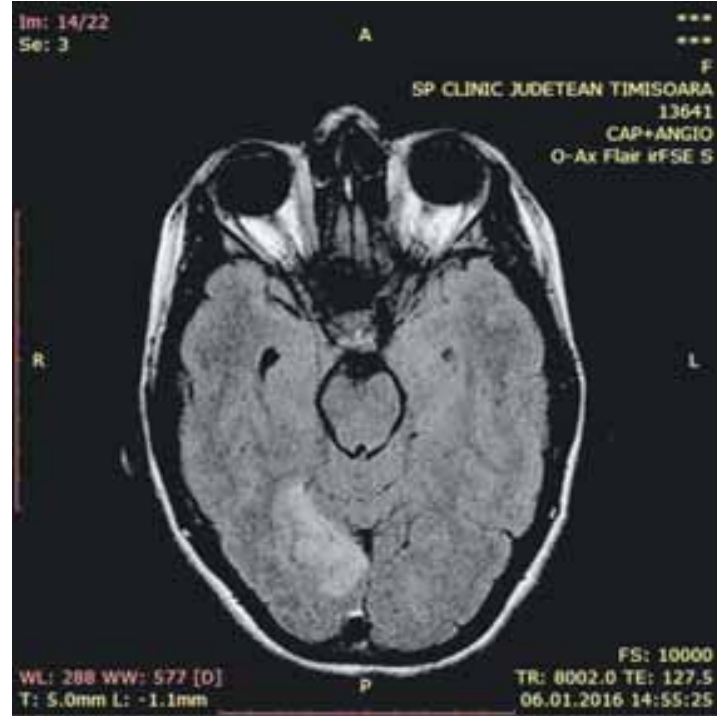

A
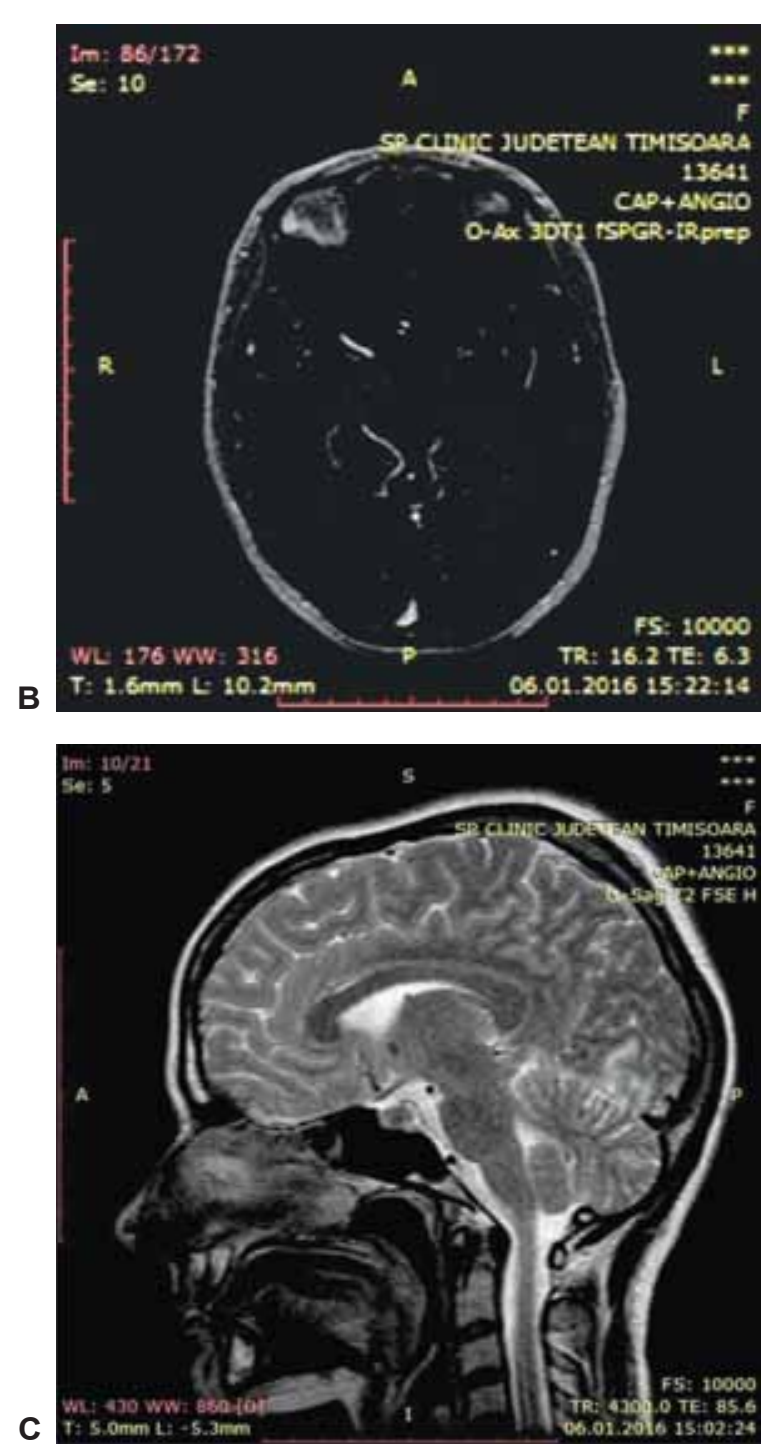

FIGURE 2. Brain contrast and non-contrast MRI revealed a cortical/subcortical lesion partially involving the right occipital lobe (hyper-signal T2 and FLAIR, slightly hypo-signal T1, no contrast uptake-suggesting an ischemic etiology for the lesion described) (Fig. $2 \mathrm{~A}$ and Fig. 2 C); brain MR-A was normal (Fig. 2 B). 
The acute clinical onset (with occipital headache and posterior cervical pain started after a sudden movement of the head) raised the suspicion of dissection of the right vertebral artery (VA). For this reason, we performed a brain and neck CTA (on the same day), which dismissed the existence of aneurysmal type dilations, as well as any kind of right VA dissection imagistic sign.

The ophthalmological exam and the automated perimetry confirmed the left superior homonymous quadrantanopia without sparing the macula (Fig. 3 $\mathrm{A}$, and $\mathrm{B})$.

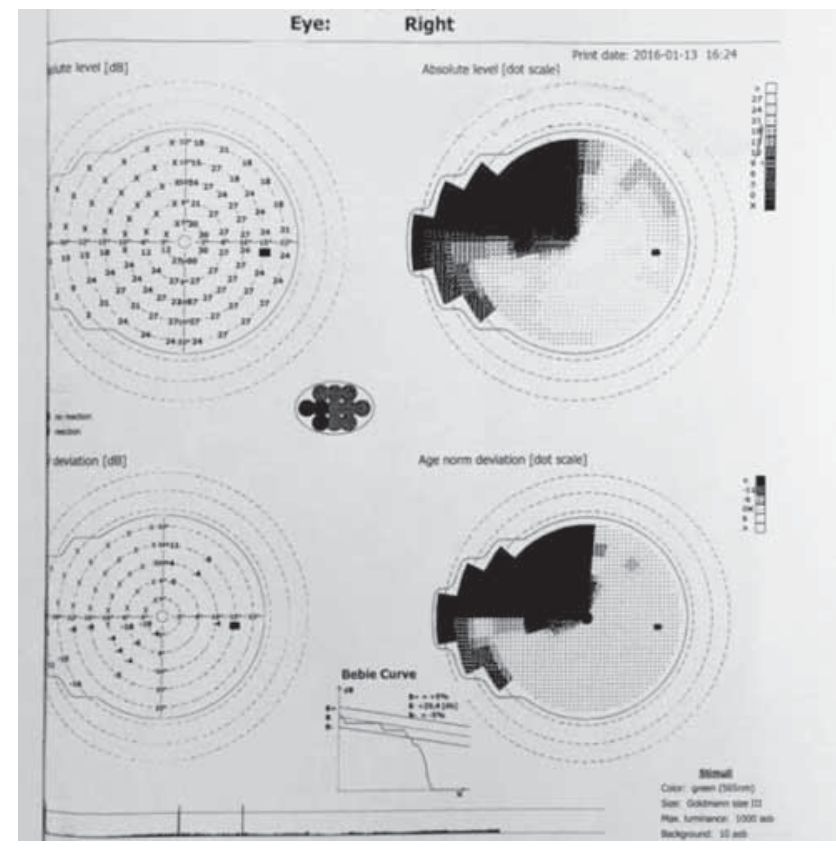

A

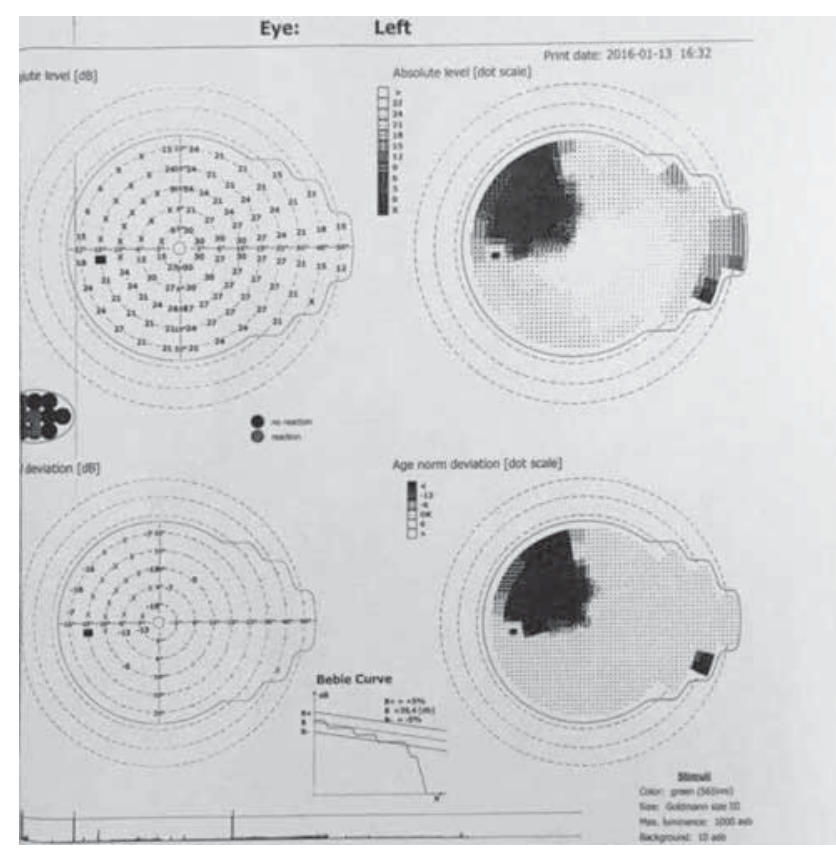

B

FIGURE 3. Automated perimetry confirming the left superior homonymous quadrantanopia without sparing the macula.
The extracranial duplex ultrasound was normal.

The electrocardiogram, chest X-ray, cardiac rhythm monitoring for 24 hours, transthoracic echocardiography (TTE) and transesophageal echocardiography (TEE) were normal and excluded the cardioembolic etiology of stroke (including foramen ovale patent).

The TEE excluded the proximal aorta etiology of ischemic stroke.

Laboratory data explored both inherited thrombophilias and acquired prothrombotic states (such as the antiphospholipid antibodies syndrome, hyper-homocysteinemia etc.), which can be associated with cerebral venous thrombosis or arterial ischemic stroke.

Laboratory tests revealed a severe inherited thrombophilia: a protein $\mathrm{S}$ deficiency $=38 \%$ (normal values $\mathrm{NV}=52.1 \%-123 \%$ for women under chronic treatment with contraceptive pills). The patient had no changes in clotting and bleeding times, aPTT, PT or INR, ESR, CRP, fibrinogen; the laboratory tests reconfirmed chronic autoimmune thyroiditis with subclinical hypothyroidism and diagnosed a mild hypercholesterolemia.

She was treated with body-weight-adjusted subcutaneous low-molecular-weight heparin (LMWH), within the first 24 hours of hospitalization, followed by oral anticoagulation with acenocoumarol, with an INR target range of 2.0 to 3.0.

The headache, vertigo and nausea resolved within two days; left superior quadrantanopia remained at discharge (after seven days). She was advised to avoid oral contraceptives, was treated with oral anticoagulants for six months of follow-up, and was instructed for permanent oral anticoagulation. We did not observe any other new stroke, deep vein thrombosis or pulmonary embolism during all this period.

\section{DISCUSSION}

Ischemic stroke resulting from nonatherosclerotic vasculopaties (including cervico-cephalic arterial dissection), large-artery atherosclerotic disease, hemodynamic mechanisms or small-vessel or penetrating artery disease (lacunes) were excluded in our case due to obviously clinical data (young age with no atherosclerotic risk factors, clinical course etc.), neuroimagistic and extracranial duplex ultrasound data (absence of extracranial and intracranial vascular disease) (5).

Ischemic stroke resulting from cardioembolism was excluded due to the absence of known prior heart disease, absence of a history of peripheral 
embolism in the limbs or abdominal viscera, absence of CT or MRI showing infarcts in multiple vascular territories. On the other hand, TTE, TEE and cardiac rhythm monitoring for 24 hours were not able to identify a potential cardiac source of emboli (5).

Head CT and MRI combined with MR venography excluded cerebral veins and dural sinus thrombosis (6).

Ischemic stroke resulting from hypercoagulable disorders (just as in our case) account for $2 \%$ to $7 \%$ of ischemic strokes in young patients (5).

The hypercoagulable conditions include the inherited thrombophilias and thrombophilias secondary to hypercoagulable states encountered in patients with malignancies, the antiphospholipid antibody syndrome (anticoagulant lupus, anticardiolipin antibodies), hyperhomocysteinemia etc (6).

Inherited thrombophilias (just as in our case) (autosomal dominant pattern of inheritance) are divided into two groups: the first group reunites the conditions caused by mutations that result in "loss of function" of anticoagulants (antithrombin deficiency, protein $\mathrm{C}$ deficiency, protein $\mathrm{S}$ deficiency just as in our case), while the second grup contains the conditions caused by mutations that result in "gain of function" of clotting factors (Factor V Leiden mutation, prothrombin gene mutation G20210A). Group 2 conditions are 5 times more frequently than Group 1 conditions, but the risk of thrombosis is higher for people with Group 1 conditions than Group 2 conditions (7).

The importance of thrombophilic disorders in arterial stroke has been debatable (8). One in seven patients with first-ever acute ischemic stroke will test positive for one of the inherited thrombophilias, but it is unlikely to be relevant to the pathogenesis of the stroke regardless of its pathogenic subtype. It was reported that there is no association between any or all of the thrombophilias combined and any of the pathogenic subtypes of ischemic stroke. Even among all patients with first-ever ischemic stroke, the prevalence of isolated thrombophilias is low and not significantly greater than in the general population $(9,10)$.

Ischemic stroke resulting from hypercoagulable disorders is a rare manifestation of protein $\mathrm{S}$ deficiency (just as in our case), the vast majority of studies revealing a weak association between the two (8). According to Mayer et al, free protein $\mathrm{S}$ deficiency $(<20 \%$ of normal total protein $S)$ is not more frequent in adults with acute cerebral infarction compared to hospitalized control patients. However, very low free protein $\mathrm{S}(<15 \%$ of normal total protein $\mathrm{S}$ ) occurred twice as frequently in patients with stroke (no statistical confirmation yet) (11).

The decision to order a thrombophilia workup can be difficult due to the inconsistent results (2). Testing for inherited thrombophilia in the setting of acute ischemic stroke is recommended if there is a history of unprovoked venous thromboembolism, venous thromboembolism in an unusual location, family history of a venous thromboembolism, thrombosis at a young age (younger than 45) and frequent thrombotic recurrences (10). Our patient had no significant pathological history or earlier clotting disorders, but we found it necessary to run these tests given the age of the patient, the use of contraceptive pills, and the absence of other causative factors of stroke.

It is important to know that many of the tests (coagulation-based studies) should not be done while patients are on anticoagulants, oral contraceptives or during pregnancy, or active thrombosis (2). Thus, the functional assays for Protein C, Protein $\mathrm{S}$ and antithrombin deficiency are affected by acute thrombosis and in the setting of anticoagulation, therefore it is recomended to repeat the assays at least 3 months after the acute thrombotic event (just as in our case, with positive results) (10).

According to Martinelli et al, oral contraceptives double the risk of ischemic stroke in the first 6-18 months of use and the presence of hyperhomocysteinemia increased the risk by 3.5 -fold, while the prothrombin gene mutation G20210A and deficiencies of antithrombin, protein $\mathrm{C}$ or protein $\mathrm{S}$ did not increase this risk (12).

\section{CONCLUSION}

Our case is an example than even if the arterial ischemic stroke is very rare in patients with protein $\mathrm{S}$ deficiency, we have to look for this association in young women, in the absence of other known causes for this type of stroke.

\section{REFERENCES}

1. Green D. Thrombophilia and stroke. Top Stroke Rehabil. 2003 Fall; 10(3):21-33.

2. Paul Schick. Hereditary and Acquired Hypercoagulability. Medscape, May 26, 2016 
3. Blickstein D., Blickstein I. Oral contraception and thrombophilia. Curr Opin Obstet Gynecol. 2007 Aug; 19(4):370-6.

4. Chisti M.M. Protein S Deficiency. Medscape, Jan 27, 2016.

5. Biller J. Chapter 36 Ischemic Cerebrovascular Disease.Practical Neurology, fourth edition, 2012 Wolters Kluwer/Lippincot Williamsand Wilkins; 385-399.

6. Piazza G. Cerebral Venous Thrombosis. Circulation. 2012; 125:17041709.

7. www.genetics.edu.au Fact Sheet 49. Blood Clotting Conditions (Hereditary Thrombophilias) Updated 15 January 2016.

8. Hooda A., Saxena P. Protein S deficiency: Recurrent ischemic stroke in young. Ann Indian Acad. Neurol. 2009 Joule-Sep; 12(3): 183-184.

9. Graeme J. Hankey G.H., Eikelboom J.W, van Bockxmeer F.M., Lofthouse E., Staples N., Baker R.I. Inherited Thrombophilia in
Ischemic Stroke and Its Pathogenic Subtypes. Stroke. 2001; 32:1793-1799.

10. Loh K.P., Ramdass S., Howard L., Saurabh Dahiya S. Association between thrombophilia and ischemic stroke: when is work-up for hypercoagulable state indicated? Clin Case Rep Rev. 2016 Volume 2(4): 380-382.

11. Mayer S.A., Sacco R.L., Hurlet-Jensen A., Shi T., Mohr J.P. Free Protein S Deficiency in Acute Ischemic Stroke. A Case-Control Sudy. Stroke. 1993; 24:224-227.

12. Martinelli I., Battaglioli T., Burgo I., Di Domenico S., Mannucci P.M. Thrombosis - Brief Report. Haematologica 2006; 91:844-847.

Conflict of interest: none declared Financial support: none declared 